\title{
Mobility of Synaptic Vesicles in Nerve Endings Monitored by Recovery from Photobleaching of Synaptic Vesicle-Associated Fluorescence
}

\author{
Kajetan Kraszewski, Laurie Daniell, Olaf Mundigl, and Pietro De Camilli \\ Department of Cell Biology and The Howard Hughes Medical Institute, Yale University School of Medicine, New Haven, \\ Connecticut 06510
}

\begin{abstract}
In nerve terminals, synaptic vesicles form large clusters anchored to the presynaptic plasmalemma. Recently, FM1-43 photobleaching experiments carried out at frog motor endplates demonstrated lack of lateral intermixing of synaptic vesicles within clusters, even during sustained nerve terminal stimulation (Henkel and Betz, 1995; Henkel et al., 1996b). We now have investigated the mobility of synaptic vesicle membranes during the endocytic limb of their exo-endocytic cycle. To this aim, we have carried out photobleaching experiments on nerve terminals of hippocampal neurons prelabeled with CY3conjugated antibodies directed against lumenal epitopes of synaptotagmin I. This conjugate is taken up specifically by synaptic vesicle membranes during endocytosis and then is recovered in newly formed synaptic vesicles. Using this method, we show that synaptic vesicle membranes intermix
\end{abstract}

after endocytosis. Staurosporine, which at hippocampal synapses partially inhibits unloading of FM1-43, but does not block uptake of antibody probes, prevents this intermixing. Our results indicate that synaptic vesicle docking and/or fusion with the plasmalemma correlate with the release of their membranes from a restraining matrix that hinders their lateral mobility. They suggest that membrane intermediates involved in synaptic vesicle reformation interact with a distinct, highly dynamic cytoskeleton and that newly formed synaptic vesicles are recaptured at random within vesicle clusters. Staurosporine, by inhibiting mobility within the terminal, may favor recapture of new vesicles near sites of endocytosis.

Key words: synapses; staurosporine; FM1-43; synaptotagmin; CY3; exocytosis; endocytosis
Synaptic vesicles are arranged in clusters under the presynaptic plasmalemma and deliver their content into the synaptic space by exocytosis (De Camilli and Jahn, 1990; Bennett and Scheller, 1994; Schweizer et al., 1995; Südhof, 1995). Rapidly after fusion, the vesicle membrane is internalized and reutilized for the regeneration of new synaptic vesicles that then are recruited into the presynaptic cluster and become available for another round of exocytosis (De Camilli and Takei, 1996). The nature of the interactions that mediate aggregation of synaptic vesicles remain poorly understood. They must be highly dynamic, because the vesicle cycle implies a translocation of vesicles from deep cytoplasmic locations to the cell surface and vice versa.

Recently, the motility of synaptic vesicles within clusters at frog motor endplates was investigated using the fluorescent dye FM1-43 (Betz et al., 1992a; Betz and Wu, 1995, Ryan and Smith, 1995). FM1-43 is a nonspecific marker for endocytic membranes. In nerve terminals, it is taken up into synaptic vesicles, which account for most of the endocytic traffic in this cellular region, and then is released by exocytosis (Betz and Bewick, 1992; Betz et al., 1992b; Ryan et al., 1993; Henkel et al., 1996a). When a small area within an FM1-43-labeled presynaptic cluster was photobleached,

\footnotetext{
Received May 2, 1996; revised July 1, 1996; accepted July 7, 1996.

This study was supported in part by grants from the Donaghue Foundation, National Institutes of Health (CA46128, DK43078), and the Human Frontier Science Program to P.D.C. We thank Drs. A. Hudson and M. Solimena for critical comments on this manuscript and L. Caron for assistance in confocal microscopy.

Correspondence should be addressed to Dr. Pietro De Camilli, Department of Cell Biology, The Howard Hughes Medical Institute, Yale University School of Medicine, 295 Congress Avenue, New Haven, CT 06510.

Copyright (C) 1996 Society for Neuroscience $0270-6474 / 96 / 165905-09 \$ 05.00 / 0$
}

no recovery from the bleaching was observed, even after nerve terminal stimulation. These findings were interpreted as indicating that synaptic vesicles within the clusters have restricted lateral mobility, although they can clearly move toward the plasmalemma to undergo exocytosis (Henkel and Betz, 1995; Henkel et al., 1996b).

Because FM1-43 is lost from vesicles with exocytosis, these studies did not allow us to demonstrate whether synaptic vesicle membranes have restricted motility in the lateral plane during the endocytic limb of the cycle (Henkel and Betz, 1995; Henkel et al., 1996b). To address this question, we now have performed photobleaching experiments in rat hippocampal neurons using a fluorescent probe that remains associated with synaptic vesicle membranes throughout the cycle: CY3-conjugated antibodies directed against the lumenal domain of synaptotagmin I (CY3-Syt lum $-\mathrm{Abs})$ (Matteoli at al., 1992; Kraszewski et al., 1995). As shown previously, these antibodies are taken up into synaptic vesicles by endocytosis and recycle in parallel with the vesicles (Matteoli et al., 1992; Mundigl et al., 1993; Kraszewski et al., 1995).

We have found that in contrast to results obtained with FM143, photobleached areas within nerve terminals labeled by CY3$\mathrm{Syt}_{\text {lum }}$-Abs recover from photobleaching after stimulation of exocytosis. This finding, combined with results of FM1-43 experiments, suggests that synaptic vesicle membranes randomly intermix during recycling. Intermixing was inhibited by the protein kinase inhibitor staurosporine (Ruegg and Burgess, 1989), in agreement with previous data demonstrating a reduction of organelle motility produced by this drug in nerve terminals (Henkel and Betz, 1995). Our results are consistent with a model in which 
a connection of synaptic vesicles to a tether that limits their lateral motility is lost after exocytosis, and in which reformed synaptic vesicles are recaptured at random into the presynaptic cluster.

\section{MATERIALS AND METHODS}

Antibodies and materials. Rabbit polyclonal antibodies directed against the lumenal domain of synaptotagmin I (Syt) were prepared, affinity purified (Mundigl et al., 1993), and conjugated to either CY3 or CY2 (Biological Detection System, Pittsburgh, PA), as described previously (Southwick et al., 1990; Kraszewski et al., 1995), or to HRP as described (Southwick et al., 1990). Tetanus toxin was the kind gift of H. Niemann (Tübingen, Germany). FM1-43 was obtained from Molecular Probes (Eugene, OR); HRP (type IV), okadaic acid, and staurosporine were obtained from Sigma (St. Louis, MO)

Hippocampal cultures. Primary cultures of hippocampal neurons were prepared from the hippocampi of 18-d-old fetal rats as described by Bartlett and Banker (1984). Cultures were used after $10 \mathrm{~d}$ in vitro.

Spot-photobleaching experiments. Neuronal cultures were briefly washed in Krebs-Ringer-HEPES (KRH) containing (in $\mathrm{mM}$ ): $128 \mathrm{NaCl}, 25$ HEPES, $4.8 \mathrm{KCl}, 1.3 \mathrm{CaCl}, 1.2 \mathrm{MgSO}_{4}, 1.2 \mathrm{KH}_{2} / \mathrm{K}_{2} \mathrm{HPO}_{4}$, and $5.6 \%$ glucose, and then incubated for $10 \mathrm{~min}$ at $37^{\circ} \mathrm{C}$ in $\mathrm{KRH} / \mathrm{high} \mathrm{K}^{+}(110 \mathrm{~mm}$ $\mathrm{K}^{+}$and a corresponding reduction in $\mathrm{Na}^{+}$) containing $3.75 \mu \mathrm{g} / \mathrm{ml} \mathrm{CY3-}$ $\mathrm{Syt}_{\text {lum }}$-Abs. In some experiments, labeling with CY3-Syt $\mathrm{lum}_{\text {lum }}$-Abs was preceded by a $1 \mathrm{hr}$ incubation with or without $2 \mu \mathrm{M}$ staurosporine in KRH. Cultures then were briefly rinsed, mounted at room temperature on the stage of an Axiovert 10 Zeiss microscope, and observed with a scanning confocal microscope (BioRad 600) equipped with $\mathrm{Kr}-\mathrm{Ag}$ laser and a Planapochromat $63 \times$ (numerical aperture, 1.4) objective. The following settings were used: $488 \mathrm{~nm}$ excitation, NDF 10\%, zoom 4.0, scan speed normal or slow. Complete spot photobleaching was performed with the command "park" while the neurons were in KRH. After spot photobleaching, neurons were incubated further (as described in Results) in $\mathrm{KRH}, \mathrm{KRH} /$ high $\mathrm{K}^{+}$, or KRH containing $2 \mu \mathrm{M}$ okadaic acid. Media were changed by a superfusion system. For each experimental condition, at least three separate experiments were performed. Average fluorescence intensity of bleached and unbleached nerve terminal areas was determined as described in the legend for Figure $1 B$.

Quantitative analysis of fluorescence probe uptake. For quantitative analysis of $\mathrm{Syt}_{\mathrm{lum}}-\mathrm{Ab}$ uptake, neurons were incubated for $10 \mathrm{~min}$ at $37^{\circ} \mathrm{C}$ in CY2-Syt lum $-\mathrm{Abs}$ in $\mathrm{KRH} /$ high $\mathrm{K}^{+}$medium, as described above for CY3-Syt ${ }_{\text {lum }}$-Abs. Then they were incubated for $1 \mathrm{hr}$ at the same temperature in KRH or KRH containing either $2 \mu \mathrm{M}$ staurosporine or $50 \mathrm{nM}$ tetanus toxin. Subsequently, they were incubated with CY3-Syt lum $_{\text {-Abs in }}$ $\mathrm{KRH}$ (either at ${ }^{\circ} 0 \mathrm{C}$ or $37^{\circ} \mathrm{C}$ ) or $\mathrm{KRH} /$ high $\mathrm{K}^{+}$. $\mathrm{FM} 1-43$ labeling was performed as described previously by Ryan et al. (1993) and Ryan and Smith (1995).

To quantify fluorescence, living neurons were observed at room temperature while in a microchamber (Warner Instrument, Hamden, CT) fitted onto the stage of a Zeiss Axiovert 35 equipped for epifluorescence (100 W mercury lamp). Fluorescence excitation and emission filters were $540 \pm 12.5$ and $590 \pm 17.5 \mathrm{~nm}$, respectively, for CY3, and $480 \pm 15$ and $530 \pm 15 \mathrm{~nm}$, respectively, for CY2 and FM1-43. Observations were performed with a chilled charged-coupled device (CCD) camera (model $\mathrm{CH}$ 250, Photometrics, Tucson, AZ) with a spatial frequency of 100 $\mathrm{nm} /$ pixel. Images were acquired and processed with an IBM computer and software (Metamorph) from Universal Imaging (West Chester, PA), stored using a digital optical disk driver (Panasonic), and printed with a video printer (Toshiba). For quantitative measurements, images were used after background subtraction. Ratios between CY3 and CY2 fluorescence were obtained by determining the average pixel brightness in a rectangle of $10 \times 10$ pixels (corresponding to $1.0 \times 1.0 \mathrm{~mm}$ ), which overlapped a fluorescent spot. For each experimental condition, measurements from at least 50 different nerve terminals from three different experiments were pooled.

Electron microscopy. Neurons were washed in KRH and then incubated for $1 \mathrm{hr}$ in $\mathrm{KRH}$ with or without staurosporine $(2 \mu \mathrm{M})$. Then they were incubated $10 \mathrm{~min}$ in $\mathrm{KRH}$ or $\mathrm{KRH} /$ high $\mathrm{K}^{+}$containing either HRP (10 $\mathrm{mg} / \mathrm{ml})$ or $\mathrm{HRP} / \mathrm{Syt}_{\text {lum }}$-Abs $(10 \mu \mathrm{g} / \mathrm{ml})$. Neurons then were fixed and processed further for electron microscopy as described previously (Mundigl et al., 1993).

\section{RESULTS}

\section{Synaptic vesicle membranes intermix during the endocytic limb of the cycle}

Synapses of cultured rat hippocampal neurons were loaded with CY3-Syt ${ }_{\text {lum }}$-Abs by incubation in the presence of the antibody conjugate in $\mathrm{KRH} / \mathrm{high} \mathrm{K}^{+}$for $10 \mathrm{~min}$. For photobleaching, elongated clusters were chosen to maximize the chance of bleaching a representative area of the vesicle cluster, i.e., an entire column of synaptic vesicles perpendicular to the plane of the presynaptic plasmalemma, rather than a layer of vesicles parallel to this membrane. Figure $1 A$, field $a$, shows a confocal microscopy image from one of such preparations immediately after antibody loading. A brightly labeled nerve terminal is visible in the field. Field $b$ shows the same area after the selective complete photobleaching (spot photobleaching) of the portion of the terminal indicated by an arrow. The spot-photobleached area appears black, whereas the surrounding area is darker than the corresponding region in field $a$ because of the fluorescence decay that occurred during image collection. This uneven distribution of the fluorescence after photobleaching persisted for as long as $30 \mathrm{~min}$ if synapses were not subjected to any further stimulation (data not shown). However, after a 3 min stimulation in $\mathrm{KRH} /$ high $\mathrm{K}^{+}$, the residual fluorescence spread homogeneously to the whole terminal with a partial recovery of fluorescence in the bleached area (field $c$ ). The spreading of the fluorescence was not attributable to phototoxicity, because the nerve terminal was able to efficiently take up FM1-43 when stimulated with $\mathrm{KRH} /$ high $\mathrm{K}^{+}$at the end of the experiment (field $d$ ). A spreading of the residual fluorescence with a partial recovery of the photobleached area also was produced by the protein phosphatase inhibitor okadaic acid (Bialojan and Takai, 1988) in the absence of depolarization (data not shown). This drug was shown previously to induce a disassembly of vesicle clusters (Betz and Henkel, 1994; Kraszewski et al., 1995, Henkel et al., 1996b).

A quantification of the images of Figure $1 A$ and of corresponding images from two other identical experiments is shown in Figure $1 B$. Bars represent ratios of fluorescence intensity between control and bleached areas of the nerve terminal in the four conditions of Figure $1 \mathrm{~A}$. Recovery from photobleaching in field $a$ is clearly illustrated by these data. These observations, which contrast with the lack of FM1-43 fluorescence spreading observed in frog motor endplates after photobleaching, have two possible interpretations. One is that the two types of synapses have different properties, and that in nerve terminals of hippocampal neurons, stimulation releases synaptic vesicles from a restraining matrix. The other is that intermixing occurs selectively during the endocytic limb of the cycle. This portion of the cycle cannot be monitored by FM1-43 photobleaching, because FM1-43 is lost from the membrane with exocytosis.

To address this issue, we repeated the FM1-43 photobleaching experiments in our culture system. Figure $2 A$, field $a$, shows a confocal image of nerve terminals loaded with FM1-43 in KRH/high $\mathrm{K}^{+}$, and field $b$ shows the same field after a portion of one of the nerve terminals has been photobleached. Field $c$ shows that a second stimulation in $\mathrm{KRH} / \mathrm{high} \mathrm{K}^{+}$leads to dimming of the unbleached area (resulting from FM1-43 unloading with synaptic vesicle exocytosis), but not to spreading of the residual fluorescence into the bleached area. Additional exposure to FM1-43 in the presence of $\mathrm{KRH} /$ high $\mathrm{K}^{+}$produced labeling of the entire nerve terminal, ruling out phototoxic effects (field $d$ ). Quantitative data from these experiments are shown in Figure $2 B$, which clearly demonstrates lack of 

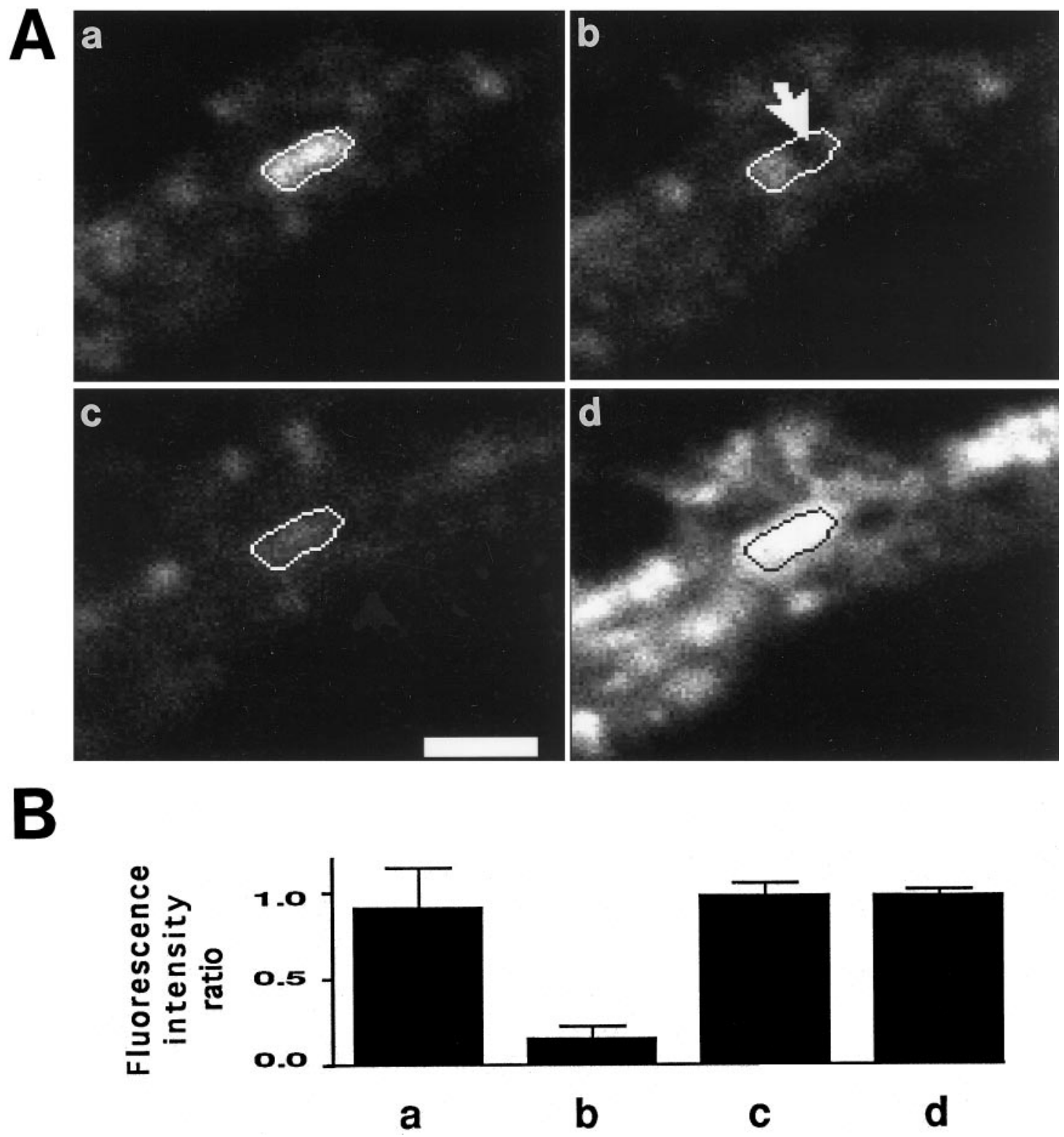

Figure 1. Effect of depolarization on the recovery from photobleaching in nerve terminals prelabeled with CY3-Syt ${ }_{\text {lum }}$-Abs. $A$, Sequential confocal microscopy images of the segment of a dendrite surrounded by axo-dendritic boutons. $a$, Presynaptic bouton after incubation with CY3-Syt ${ }_{\text {lum }}$-Abs for $10 \mathrm{~min}$ in $\mathrm{KRH} / \mathrm{high} \mathrm{K}^{+}$. The fluorescent area surrounded by a dotted white line represents a presynaptic terminal filled with fluorescent synaptic vesicles. The same field is shown in $b$ after complete laser-induced spot photobleaching of the portion of the terminal indicated by an arrow. The same field is shown in $c$ after a second 3 min depolarization with $\mathrm{KRH} / \mathrm{high} \mathrm{K}^{+}$, and in $d$ after an incubation for 5 min with FM1-43 in $\mathrm{KRH} /$ high $\mathrm{K}^{+}$. The last incubation represents a control to show that the bleached nerve terminal still is fully viable and takes up FM1-43. A partial global loss of CY3 fluorescence from one field to the next is attributable to fluorescence decay during image collection. Note that in $c$, the residual fluorescence spreads to the whole nerve terminal, resulting in a partial recovery of fluorescence in the photobleached area. Scale bar, $2 \mu \mathrm{m}$. B, Quantification of results obtained from the experiment shown in $A$ and from two additional similar experiments. Bars represent ratios of fluorescence intensity between the control region of the terminal and the region subjected to photobleaching. Fluorescence intensity was measured with a confocal microscope on $0.16 \mu \mathrm{m}^{2}(36$ pixels) fields corresponding to the central areas of each of the two nerve terminal regions. Error bars represent SD.

recovery from photobleaching after depolarization. We conclude that even in presynaptic nerve terminals of CNS neurons, translocation of synaptic vesicles to the presynaptic plasmalemma occurs without a substantial intermixing in the lateral plane. Thus, the spreading of fluorescence observed with CY3-Syt lum $_{\text {-Abs must result }}$ from membrane intermixing during synaptic vesicle reformation. This conclusion is corroborated further by the observation that recovery from photobleaching in nerve terminals labeled with CY3$\mathrm{Syt}_{\text {lum }}$-Abs was not observed if neurons were treated with tetanus toxin before the stimulation in $\mathrm{KRH} / \mathrm{high} \mathrm{K}^{+}$(data not shown). This toxin (Schiavo et al., 1992; Blasi et al., 1993) patently blocks synaptic vesicle exocytosis in cultured hippocampal neurons (Mundigl et al., 1995). Thus, synaptic vesicle exocytosis is required for synaptic vesicle-membrane intermixing after nerve terminal depolarization.

\section{Staurosporine blocks synaptic vesicle-membrane intermixing}

It was reported that after treatment with the protein kinase inhibitor staurosporine (Ruegg and Burgess, 1989), evoked release of neurotransmitter is not accompanied by a corresponding 

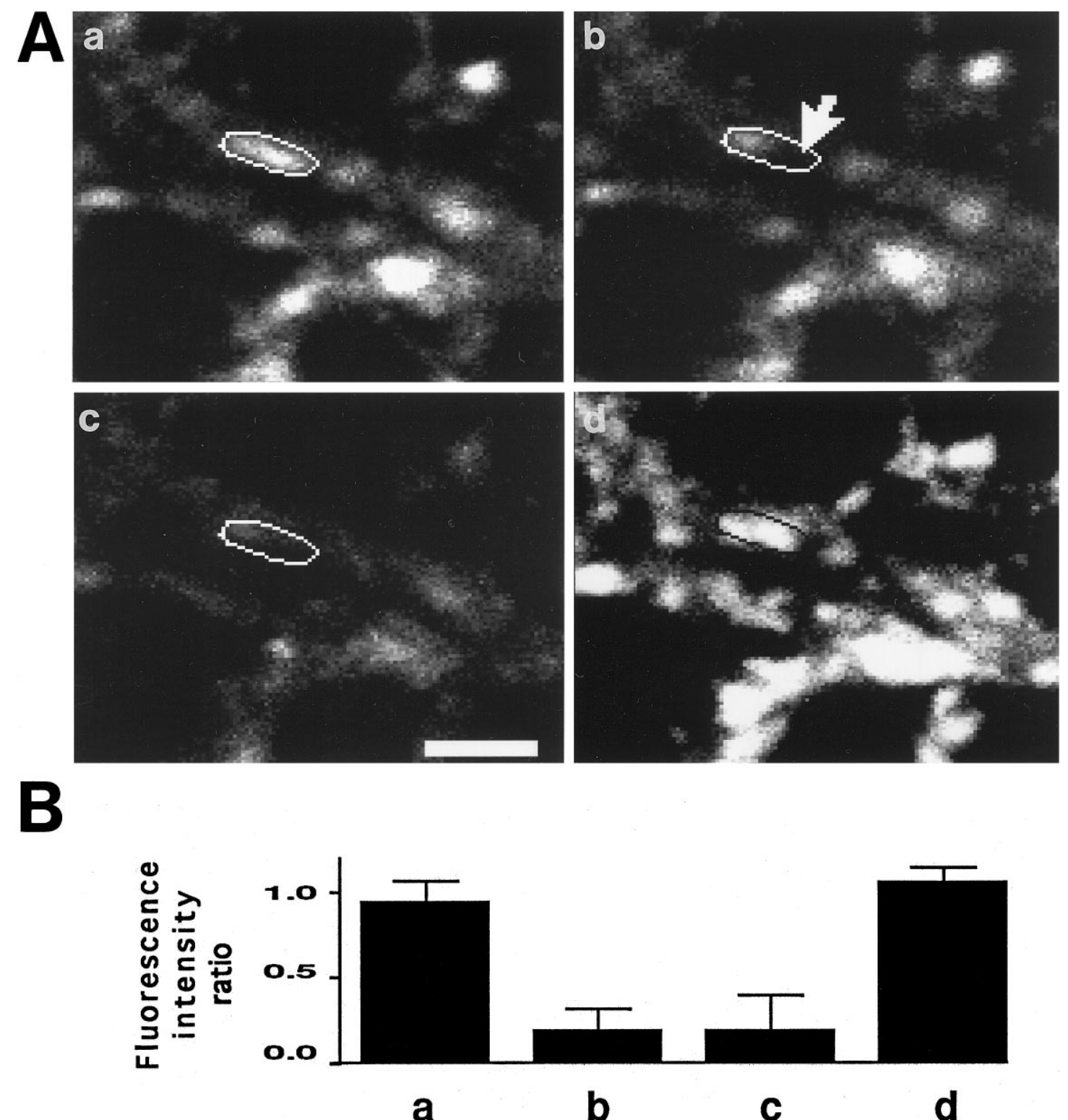

Figure 2. Photobleached areas in FM1-43-labeled nerve terminals do not recover from photobleaching after depolarization. $A$, Sequential confocal microscopy images of nerve terminals labeled with FM1-43. The same field is shown in $a$ after loading with FM1-43 in KRH/high $\mathrm{K}^{+}$for 10 min, in $b$ after photobleaching of one nerve terminal, in $c$ after 3 min depolarization with $\mathrm{KRH} / \mathrm{high} \mathrm{K}^{+}$, and in $d$ after a new loading with $\mathrm{FM} 1-43$ in $\mathrm{KRH} / \mathrm{high} \mathrm{K}^{+}$ Note that in $c$, the unbleached part is significantly dimmer than in $b$ (attributable to FM1-43 release with exocytosis and to previous image collection), but there is no diffusion of fluorescent signal from the unbleached to the bleached portion of the terminal. Scale bar, $2 \mu \mathrm{m}$. $B$, Quantification of results obtained from the experiment shown in $A$ and from two additional similar experiments (see legend to Fig. 1B).

release of FM1-43 (Henkel and Betz, 1995). This property of staurosporine has been exploited to determine whether FM1-43 fluorescence recovers from photobleaching after stimulation. In contrast to our results obtained with CY3-Syt ${ }_{\text {lum }}$-Abs, no intermixing was observed (Henkel and Betz, 1995).

We examined therefore whether in hippocampal neurons staurosporine affected recovery from photobleaching after CY3-Syt $\mathrm{lum}_{\text {lum }}-\mathrm{Ab}$ labeling. Figure 3 illustrates the results obtained when hippocampal cultures were subjected to the same experimental protocol used for Figure 1, but after the neurons had been pretreated for $1 \mathrm{hr}$ in the presence of staurosporine $(2 \mu \mathrm{M})$. Figure $3 A$, field $a$, shows a confocal image of two nerve terminals at the end of the load with CY3$\mathrm{Syt}_{\text {lum }}$-Abs. Field $b$ shows the same field after partial photobleaching of two the nerve terminals, field $c$, after an additional depolarization with $\mathrm{KRH} /$ high $\mathrm{K}^{+}$for $3 \mathrm{~min}$, and field $d$ after a control uptake of FM1-43. Comparison of field $b$ with field $c$ indicates that staurosporine blocks diffusion of fluorescent signal from unbleached to bleached portions of the nerve terminal. Quantification of these results is shown in $B$. We thus tentatively conclude that staurosporine greatly limits the motility within terminals of synaptic vesicle membranes during recycling. Staurosporine pretreatment also completely blocked the recovery of photobleaching produced by okadaic acid (data not shown), in agreement with results of Henkel et al. (1996b).

\section{Staurosporine does not block uptake of Syt lum $_{\text {-Abs }}$ during exo-endocytosis}

An additional implication of the experiments illustrated in Figure 3 is that staurosporine treatment does not block nerve terminal 

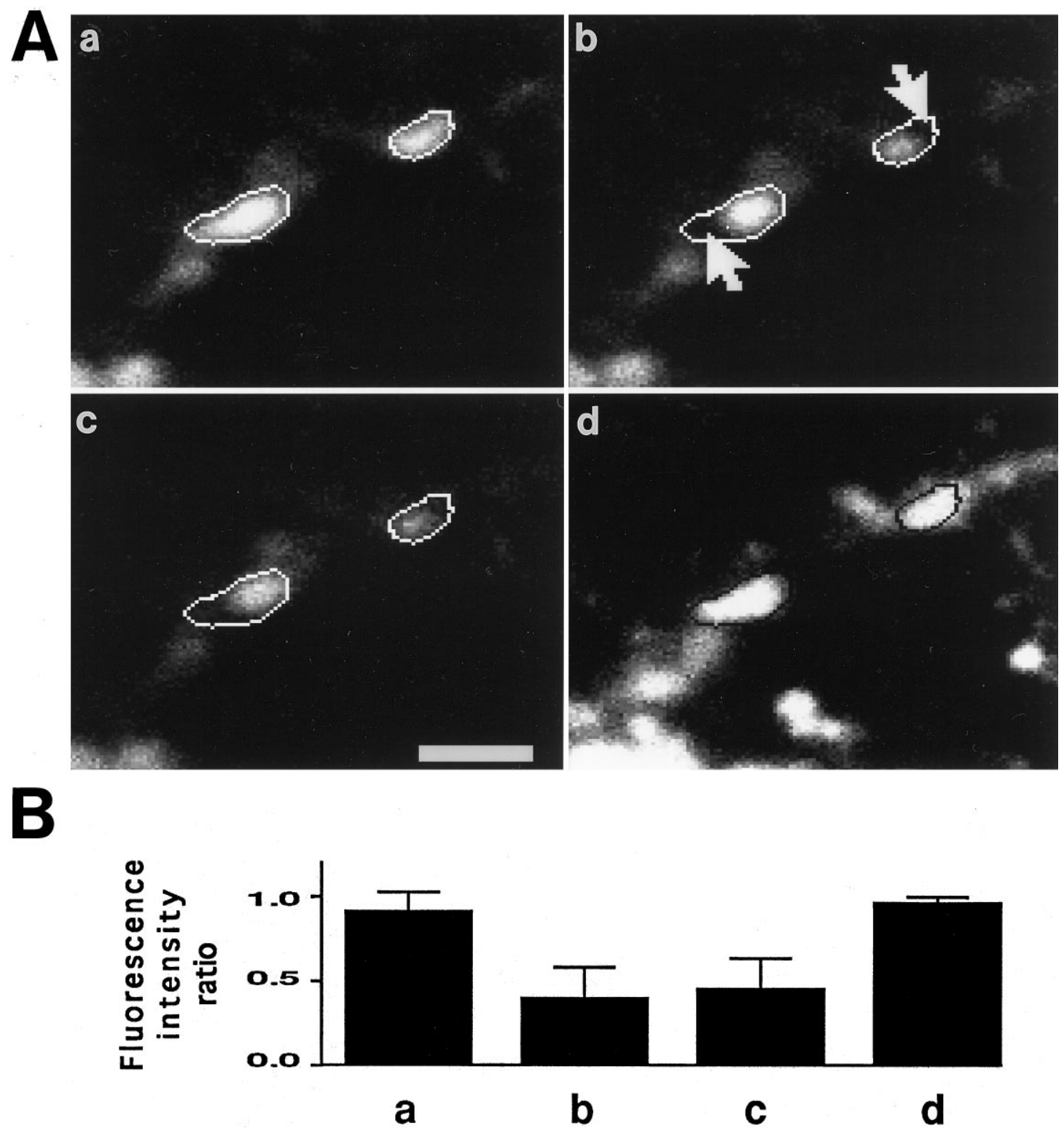

Figure 3. Staurosporine blocks depolarization-induced recovery from photobleaching in neurons labeled with CY3-Syt lum $^{-A b s . ~} A$, Sequential confocal microscopy images of nerve terminals labeled with CY3-Syt ${ }_{1 u m}$-Abs. Nerve terminals were incubated for $1 \mathrm{hr}$ with staurosporine $(2 \mu \mathrm{M})$ and then exposed

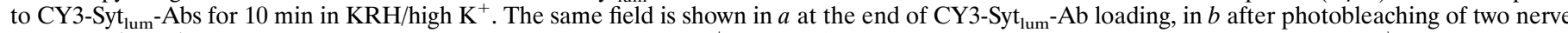
terminals (arrow), in $c$ after 3 min depolarization with $\mathrm{KRH} / \mathrm{high} \mathrm{K}^{+}$, and in $d$ after loading with FM1-43 for 5 min in $\mathrm{KRH} /$ high $\mathrm{K}^{+}$to show that the bleached nerve terminals still are fully viable and take up the dye. Note that in $c$, the residual fluorescence does not spread to the bleached area of nerve terminals with depolarization. Scale bar, $2 \mu \mathrm{m}$. $B$, Quantification of results obtained from the experiment shown in $A$ and from two additional similar experiments (see legend to Fig. $1 B$ ).

loading with CY3-Syt lum -Abs. Henkel and Betz (1995) reported that staurosporine, in addition to blocking release of FM1-43, also blocks the uptake of fluid-phase HRP at the frog neuromuscular junction. These findings were taken as an indication that after treatment with staurosporine, exocytosis occurs via a narrow, extremely transient fusion pore. The properties and opening time of the pore would prevent significant diffusion of macromolecules and unloading of FM1-43, a membrane-bound substance (Betz and Bewick, 1992), which is not as rapidly releasable as soluble neurotransmitters (Henkel and Betz, 1995). The efficient uptake of CY3-Syt ${ }_{\text {lum }}$-Abs in staurosporine-treated nerve terminals (Fig. $3 A$, field $a$ ) was in apparent contrast with these previous results, because antibody conjugates are larger than HRP. We examined therefore whether staurosporine also blocks FM1-43 release in synapses of hippocampal cultures. Neurons were incubated for 1 $\mathrm{hr}$ in the presence or absence of staurosporine $(2 \mu \mathrm{M})$ and then loaded with FM1-43 in KRH/high $\mathrm{K}^{+}$. Subsequently, the effect of $\mathrm{KRH} /$ high $\mathrm{K}^{+}$on FM1-43 unloading was measured. As shown in Figure 4, staurosporine inhibited FM1-43 release, although the inhibition was not as strong as observed previously at the frog motor endplate after electrical stimulation (Betz and Bewick, 1992).

We considered the possibility that an inhibitory effect of staurosporine on $\mathrm{Syt}_{\mathrm{lum}}$-Ab uptake may be detected by a quantitative assay. To perform an accurate comparison between the uptake of antibodies before and after staurosporine treatment, we developed a double-labeling technique based on Syt $_{1 u m}$-Abs conjugated 


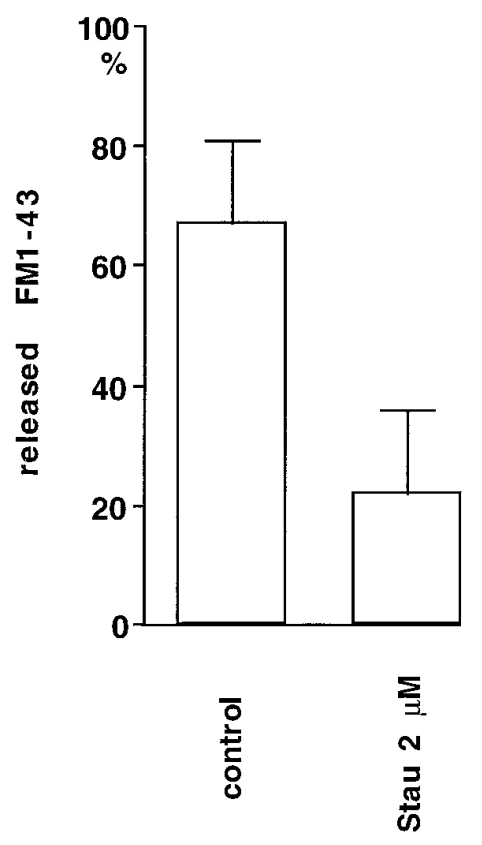

Figure 4. Staurosporine blocks the unloading of FM1-43. Neurons were incubated $1 \mathrm{hr}$ in $\mathrm{KRH}$ with or without staurosporine $(2 \mu \mathrm{M})$, loaded with FM1-43 in $\mathrm{KRH} / \mathrm{high} \mathrm{K}^{+}$for $10 \mathrm{~min}$, rinsed in $\mathrm{KRH}$, and then reexposed to $\mathrm{KRH} / \mathrm{high} \mathrm{K}^{+}$in the absence of FM1-43 to unload the dye. Bars indicate ratios between the fluorescence intensity observed on individual boutons at the end of the load and after the unloading period. Fluorescence intensity was measured with a chilled CCD camera. Error bars represent SEM.

to two different fluorochromes with different emission characteristics, CY3 and CY2 (Southwick et al., 1990). A similar method based on Syt $_{\text {lum }}$-Abs raised in two different species was applied previously to determine the presynaptic contribution of long-term potentiation (Malgaroli et al., 1995).

Neurons first were exposed to a control incubation with CY2$\mathrm{Syt}_{\text {lum }}$-Abs. Then, after an incubation in KRH with or without further additions, they were incubated with CY3-Syt lum $_{\text {-Abs. The }}$ $\mathrm{CY} 3 / \mathrm{CY} 2$ fluorescence ratio, measured with a CCD camera, provided an indication of the difference in the exo-endocytotic rate between the second and first incubations. In pilot experiments, we determined that the same CY3/CY2 ratio was observed irrespectively of the order with which the two labels were applied. The same ratio also was observed when the two conjugates were applied simultaneously, indicating that antibody binding is far from saturation and that labeling with one conjugate does not affect labeling with the other.

The effectiveness of this method was determined by control experiments, as illustrated in Figure 5. Bar $a$ shows the CY3/CY2 ratios obtained when both the first and second incubations were performed for $10 \mathrm{~min}$ in $\mathrm{KRH} / \mathrm{high} \mathrm{K}^{+}$and the intervening incubation ( $1 \mathrm{hr})$ in $\mathrm{KRH}$. This ratio, defined as $100 \%$, was much higher than the ratio observed when the second incubation was performed in normal KRH (bar $b$ ), in agreement with the potent stimulatory effect on synaptic vesicle exocytosis of $\mathrm{KRH} / \mathrm{high}^{+}$. An even lower ratio was observed when the second incubation was performed in $\mathrm{KRH}$ at $0^{\circ} \mathrm{C}$ (bar $c$ ), i.e., a condition in which exo-endocytosis is blocked. A very similar low ratio was observed when neurons were treated with tetanus toxin between the first and second incubations (bar $d$ ).

The effect of staurosporine on $\mathrm{Syt}_{1 \mathrm{um}}-\mathrm{Ab}$ uptake as determined

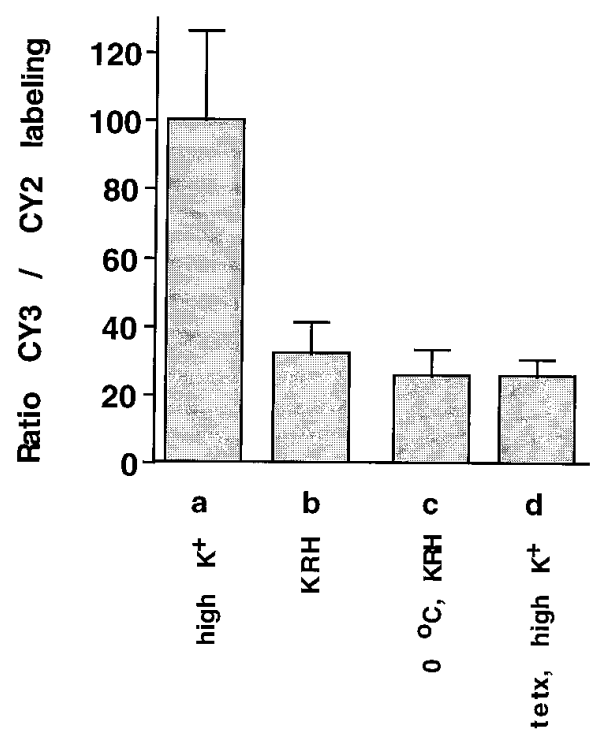

Figure 5. Quantitative analysis of exo-endocytosis based on the comparative uptake of CY3-Syt $\mathrm{lum}_{\mathrm{um}}$-Abs and CY2-Syt ${ }_{\mathrm{lum}}$-Abs. Neurons first were incubated with CY2-Syt lum $^{-A b s}$ for $10 \mathrm{~min}$ in $\mathrm{KRH} / \mathrm{high} \mathrm{K}^{+}$. Then after one additional hour in KRH, which contained tetanus toxin $(50 \mathrm{nM})$ in the case of test condition $d$, they were incubated with CY3-Syt lum $^{-A b s}$ for 10 min under the following test conditions: $\mathrm{KRH} / \mathrm{high} \mathrm{K}^{+}$in $a, \mathrm{KRH}$ in $b$, $\mathrm{KRH}$ at $0^{\circ} \mathrm{C}$ in $c$, and $\mathrm{KRH} /$ high $\mathrm{K}^{+}$in $d$. Bars represent the ratio between the CY3 and CY2 fluorescence (which was measured at the end of the experiments with a chilled CCD camera) expressed as percentages of the $\mathrm{CY} 3 / \mathrm{CY} 2$ ratio observed in the test condition $a$. Error bars represent SEM.

by this assay is illustrated in Figure 6 . The figure illustrates the CY3/CY2 ratios observed when a 10 min loading with CY2Syt $_{\text {lum }}$-Abs was followed by $1 \mathrm{hr}$ incubation with or without staurosporine $(2 \mu \mathrm{M})$ and then by loading with CY3-Syt lum $_{\text {-Abs }}$ for the times indicated. Staurosporine did not have a significant inhibitory effect on $\mathrm{Syt}_{\mathrm{lum}}-\mathrm{Ab}$ uptake. Thus, this protein kinase inhibitor does not appear to reduce synaptic vesicle-membrane availability for antibody binding at the cell surface during the exo-endocytosis.

To further confirm that $\mathrm{Syt}_{1 \mathrm{um}}$-Abs are taken up into synaptic vesicles even after staurosporine treatment, electron microscopy was performed. Syt $\mathrm{Sum}_{\text {lum }}$-Abs were conjugated to HRP, and neurons were incubated with the antibody conjugates for $10 \mathrm{~min}$ after a 1 hr pretreatment with either KRH or KRH containing staurosporine $(2 \mu \mathrm{M})$. In both cases, HRP reaction product was found in synaptic vesicles, and the fraction of labeled vesicles was similar in the two conditions (Fig. 7). The average fraction of labeled synaptic vesicles per cross-section of the nerve terminal was $7.8 \pm$ $4.4 \%$ in control neurons $(a)$ and $6.6 \pm 4.8 \%$ in staurosporinetreated neurons $(b)$. The same results were obtained when neurons were exposed to fluid-phase HRP. The fraction of labeled vesicles per nerve terminal cross-section was $18.3 \pm 4.6 \%$ in control neurons $(c)$ and $18.9 \pm 5.3 \%$ labeled vesicles in staurosporine-treated neurons $(d)$. These results are in full agreement with data obtained by quantitative videomicroscopy and indicate that in hippocampal synapses, staurosporine does not block uptake of protein markers.

Analyses of electron micrographs from neurons treated with $\mathrm{HRP} / \mathrm{Syt}_{\text {lum }}$-Abs or fluid-phase HRP also demonstrated that labeled synaptic vesicles were randomly interspersed within the vesicle cluster. This observation speaks against the possibility that 


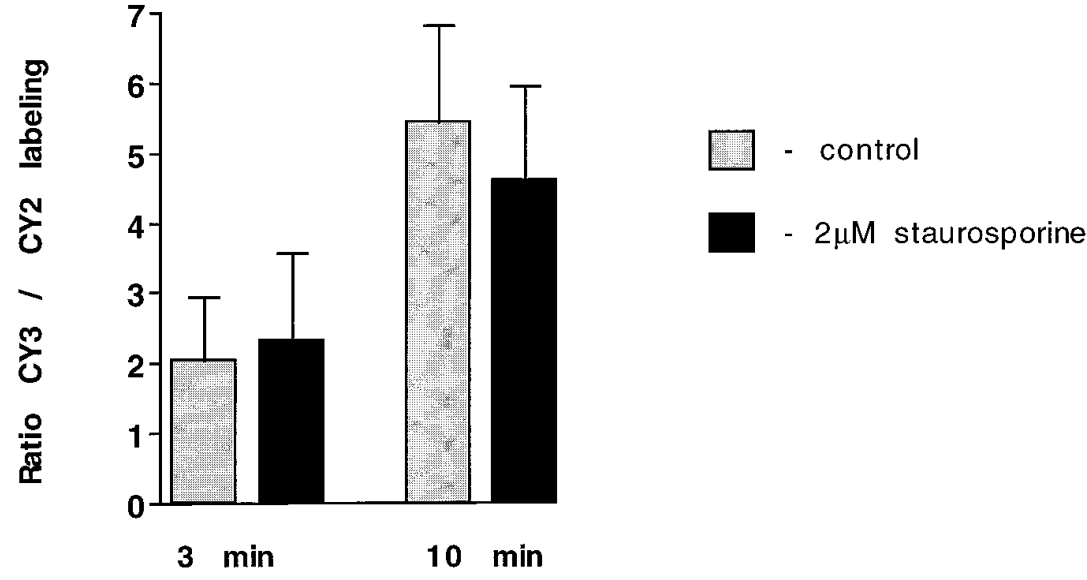

Figure 6. Staurosporine does not block the uptake of CY3-Syt ${ }_{\text {lum }}-$ Abs. Neurons were incubated in $\mathrm{KRH} /$ high $^{+}$ containing CY2-Syt ${ }_{\text {lum }}-\mathrm{Abs}$ for $10 \mathrm{~min}$, then for $1 \mathrm{hr}$ in $\mathrm{KRH}$ with or without staurosporine $(2 \mu \mathrm{M})$, and finally for 3 or 10 min with $\mathrm{KRH} / \mathrm{high}^{+}$containing CY3-Syt ${ }_{\text {lum }}$-Abs. Bars represent ratios between the CY2 and CY3 fluorescence at the end of the experiment. after staurosporine, only a small fraction of synaptic vesicles, for example, the pool of vesicles closer to the presynaptic plasmalemma, participate in stimulated exo-endocytosis.

\section{DISCUSSION}

The results of our study are consistent with a model in which synaptic vesicles have a restricted lateral mobility within presynaptic vesicle clusters (Henkel and Betz, 1995, Henkel et al., 1996b). However, they demonstrate that during recycling, newly reformed synaptic vesicles are incorporated in the cluster at random in the lateral plane. This intermixing is inhibited by staurosporine.

The restricted lateral mobility of synaptic vesicles within presynaptic vesicle clusters was demonstrated previously in FM1-43 photobleaching experiments at the frog neuromuscular junction
(Henkel and Betz, 1995; Henkel et al., 1996b). Our present study extends this conclusion to CNS synapses in cultures, and complements it with information on the mobility of synaptic vesicles during the endocytic limb of their cycle. These new data have been obtained by the combined use of FM1-43, which labels synaptic vesicles through a single cycle (Betz and Bewick, 1992; Henkel et al., 1996a; Ryan et al., 1996), and Syt $_{1 \text { lum }}$-Abs, which remain associated with synaptic vesicles membranes through multiple cycles of exo-endocytosis (Matteoli et al., 1992; Kraszewski et al., 1995).

In presynaptic nerve terminals, the reserve pool of synaptic vesicles are clustered together behind a layer of docked vesicles (Couteaux and Pecot-Dechavassine, 1974). The partial resistance of these clusters to homogenization (Navone et al., 1984) and the
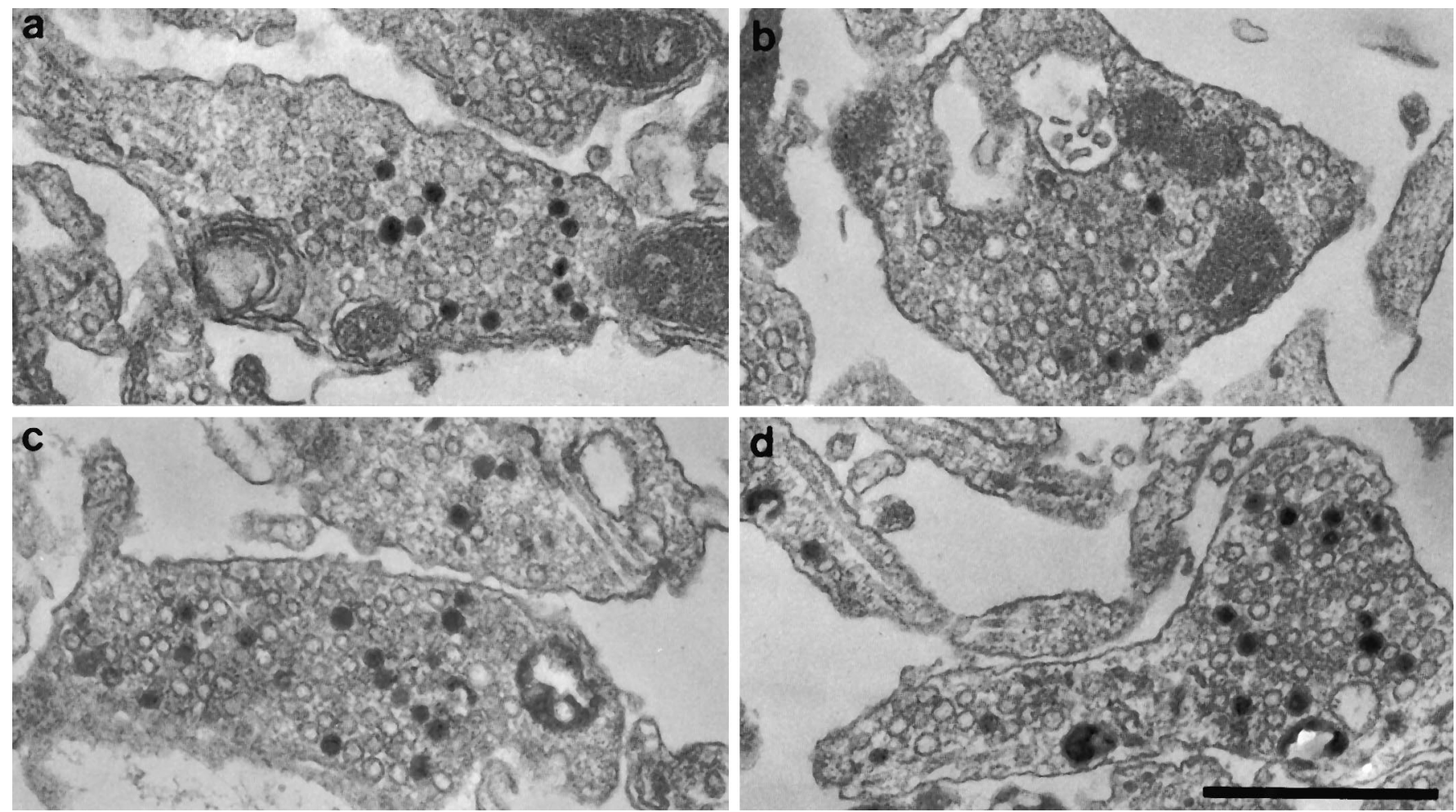

Figure 7. Staurosporine does not block uptake of both HRP/Syt ${ }_{\text {lum }}-\mathrm{Abs}$ and HRP in nerve terminals of hippocampal neurons. Electron micrographs of nerve terminals from cultures incubated first for $1 \mathrm{hr}$ in $\mathrm{KRH}$ with $(b, d)$ or without $(a, c)$ staurosporine $(2 \mu \mathrm{M})$, then for $10 \mathrm{~min}$ with $\mathrm{HRP} / \mathrm{Syt}_{\mathrm{lum}}$-Abs $(a, b)$ or HRP $(c, d)$ in $\mathrm{KRH} / \mathrm{high} \mathrm{K}^{+}$. Note presence of HRP-labeled synaptic vesicles in all conditions. 
direct electron microscopic demonstration of a trabecular network interconnecting the vesicles (Landis et al., 1988; Hirokawa et al., 1989) suggest that formation of the clusters is mediated by a cytoskeletal matrix to which vesicles are tethered, and which may include actin (Hirokawa et al., 1989) and synapsin (De Camilli et al., 1983, 1990; Pieribone et al., 1995; Rosahl et al., 1995). Lack of recovery from FM1-43 photobleaching even in a stimulated nerve terminal (Henkel and Betz, 1995, Henkel et al., 1996b; present study) strongly suggests that vesicles retain an association with this cytomatrix as they translocate to the presynaptic plasmalemma (Henkel and Betz, 1995).

Synaptic vesicle intermixing after exocytosis implies that this tether is lost with vesicle fusion. Mechanisms of synaptic vesicles reformation are not fully understood, but strong evidence indicates a key role of the clathrin coat (Heuser and Reese, 1973; Pfeffer and Kelly, 1985; Maycox et al., 1992; Takei et al., 1996). Clathrin-coated vesicles and pits generally are localized at the periphery of synaptic vesicle clusters or even at some distance from them (Heuser and Reese, 1973; Miller and Heuser, 1984; Harris and Sultan, 1995; Takei et al., 1996). It is therefore plausible that they may not be attached to the same cytomatrix that interconnects synaptic vesicles to each other, and that a recycling synaptic vesicle membrane may become trapped by this cytomatrix only after it has been modified to become a new mature synaptic vesicle. We have shown recently that after depolarization with high $\mathrm{K}^{+}$, a fraction of synaptic vesicles reform via clathrinmediated budding from endosome-like structures that in turn derive from deep invaginations of the plasmalemma (Takei et al., 1996) (see also Fried and Blaustein, 1978; Miller and Heuser, 1984). Because these endosome-like intermediates also are excluded from synaptic vesicle clusters, and often are located deeply in the nerve terminal cytoplasm (Miller and Heuser, 1984; Takei et al., 1996), their intracellular mobility may contribute to membrane intermixing during recycling.

The block of synaptic vesicle-membrane intermixing by staurosporine complements results obtained by Henkel and Betz (1995). These authors also reported other effects of staurosporine. They showed that after staurosporine treatment, release of neurotransmitter from motor endplates is neither accompanied by loading of recycling synaptic vesicles with HRP nor by unloading of FM1-43 (this lack of unloading was the basis for their demonstration that synaptic vesicles do not intermix during endocytosis in staurosporine-treated nerve terminals). To explain these data, they proposed that staurosporine modifies the properties of the exocytotic process; after treatment with this drug, neurotransmitter release would occur via a very narrow and transient fusion pore. Although the water-soluble neurotransmitter could diffuse rapidly through the pore, neither macromolecules such as HRP nor membrane-bound compounds such as FM1-43 could diffuse through this opening. Unloading of FM1-43 during exoendocytosis requires either dissociation from the membrane (a process that occurs with the half-time of seconds [Ryan and Smith, 1995]) or diffusion away from the exocytotic pit before pore closure. Fast closure of the pore would prevent both events (Henkel and Betz, 1995).

In the present study, we have found also that at hippocampal synapses, staurosporine partially inhibits FM1-43 release in addition to preventing vesicle intermixing during recycling. However, we have not observed a major effect of the drug on the uptake of macromolecules, such as $\mathrm{Syt}_{1 \mathrm{um}}$-Abs or of fluid-phase HRP. We cannot exclude that this discrepancy may be explained by the different experimental systems, because the data of Henkel and
Betz (1995) were obtained at the frog neuromuscular junction rather than at CNS synapses, and with electrical stimulation rather than with $\mathrm{K}^{+}$-induced depolarization. Our observations, however, show that in hippocampal neurons, staurosporine blocks synaptic vesicle-membrane intermixing and partially inhibits FM1-43 release under conditions in which macromolecule uptake is not significantly affected. They challenge therefore the interpretation that staurosporine affects size and opening time of the exocytotic pore. We suggest that the inhibitory effect of staurosporine on vesicle intermixing may be mediated by an inhibition of organelle mobility in nerve terminals, possibly attributable to effects of the drug on the cytoskeleton. For example, it was reported that staurosporine inhibits motility of FM1-43-labeled endosomes (Henkel and Betz, 1995). A reduced mobility of clathrin-coated vesicles and endosomal intermediates may enhance the probability that a newly formed vesicle is recaptured locally within the cluster.

The inhibitory effect of staurosporine on FM1-43 release remains very puzzling and of difficult interpretation. Increasing evidence suggests that vesicle fusion and budding may correlate with metabolic changes in lipid components of the membranes (De Camilli et al., 1996). It cannot be excluded that retention of FM1-43 after staurosporine treatment may reflect changes produced by this protein kinase inhibitor on membrane lipids that may modify the rate of dissociation of the dye from membranes. Indirect effects of staurosporine, mediated by a general reduced motility of the submembranous cytoskeleton, also may play a role in this phenomenon.

Recent FM1-43 studies have indicated that the bulk of synaptic vesicles present in nerve terminals of cultured CNS neurons undergo recycling within the first minute of depolarizing with KRH/high K ${ }^{+}$(Ryan and Smith, 1995). Labeling of only a fraction of synaptic vesicles by either HRP or $\mathrm{Syt}_{\text {lum }}$-Abs after $10 \mathrm{~min}$ incubations in $\mathrm{KRH} /$ high $\mathrm{K}^{+}$(Fig. 7) probably is explained by the low probability of these macromolecular probes to enter a vesicle at the protein concentrations that typically are used for these experiments. In this study, the concentrations of soluble HRP and $\mathrm{HRP} /$ Syt $_{\text {lum }}$-Abs were $10 \mathrm{mg} / \mathrm{ml}$ and $10 \mu \mathrm{g} / \mathrm{ml}$, respectively. These concentrations are much lower (on a molar basis) than the concentrations that can be achieved with FM1-43. Note that a similar proportion of labeled vesicles can be obtained (Fig. 7) with a 1000 -fold lower concentration of $\mathrm{Syt}_{\text {lum }}$-Abs (a high-affinity ligand for the inner face of the vesicle) than with free HRP (a fluid-phase marker). The proportion of vesicles that can be labeled by Syt $_{l_{u m}}{ }^{-}$ Abs was found to increase by increasing the concentration of antibodies (O. Mundigl, L. Daniell, P. De Camilli, unpublished observations), thus indicating that the partial labeling of the synaptic vesicle population by these probes is not attributable to two qualitatively different types of exo-endocytotic events. A single peroxidase molecule is sufficient to generate reaction product in a small vesicle (Stinchcombe et al., 1995), and therefore is possible that labeled synaptic vesicles may reflect presence in the lumen of a single enzyme molecule.

In conclusion, our results provide some new information on the dynamics of synaptic vesicles in nerve terminals. They indicate that interactions of the vesicle membrane with the cytomatrix change as the vesicle proceeds through its cycle. They demonstrate major differences in the mechanisms that translocate vesicles toward, and away from, the presynaptic plasmalemma, and indicate a much greater vesicle membrane motility during the endocytic limb of the cycle. They are consistent with a model in which synaptic vesicles do not reform directly from the site of 
exocytosis to be recaptured immediately in the presynaptic cluster. They indirectly support a participation of clathrin-coated vesicles and endosome-like intermediates in synaptic vesicle recycling, as suggested by other studies (Miller and Heuser, 1984; Takei et al., 1996).

\section{REFERENCES}

Blasi J, Chapman ER, Yamasaki S, Binz T, Niemann H, Jahn R (1993) Botulinum neurotoxin $\mathrm{C} 1$ blocks neurotransmitter release by means of cleaving HPC-1/syntaxin. EMBO J 12:4821-4828.

Bartlett WP, Banker GA (1984) An electron microscopic study of the development of axon and dendrites by hippocampal neurons in culture. I. Cells which develop without intracellular contacts. J Neurosci 4:1944-1953.

Bennett MK, Scheller RH (1994) A molecular description of synaptic vesicles membrane trafficking. Annu Rev Biochem 63:63-100.

Betz WJ, Bewick GS (1992) Optical analysis of synaptic vesicle recycling at the frog neuromuscular junction. Science 255:200-203.

Betz WJ, Henkel AW (1994) Okadaic acid disrupts clusters of synaptic vesicles in frog motor nerve terminals. J Cell Biol 124:843-854.

Betz WJ, Wu LG (1995) Kinetics of synaptic vesicle recycling. Curr Biol 5:1102-1105.

Betz WJ, Bewick GS, Ridge RM (1992a) Intracellular movements of fluorescently labeled synaptic vesicles in frog motor nerve terminals during nerve stimulation. Neuron 9:805-813.

Betz WJ, Mao F, Bewick GS (1992b) Activity dependent fluorescent staining and destaining of living vertebrate motor nerve terminals. J Neurosci 12:363-375.

Bialojan C, Takai A (1988) Inhibitory effect of a marine-sponge toxin, okadaic acid, on protein phosphatases: specificity and kinetics. Biochem J 256:283-290.

Couteaux R, Pecot-Dechavassine M (1974) Les zones specialisees des membranes presynaptiques. Comptes Rendus Hebdomadaires des Seances de l'Academie des Sciences D 278:291-293.

De Camilli P, Jahn R (1990) Pathways to regulated exocytosis in neurons. Annu Rev Physiol 52:625-645.

De Camilli P, Takei K (1996) Molecular mechanisms in synaptic vesicle endocytosis and recycling. Neuron 16:481-486.

De Camilli P, Harris Jr SM, Huttner WB, Greengard P (1983) Synapsin I (protein I), a nerve terminal-specific phosphoprotein. II. Its specific association with synaptic vesicles demonstrated by immunocytochemistry in agarose-embedded synaptosomes. J Cell Biol 96:1355-1373.

De Camilli P, Benfenati F, Valtorta F, Greengard P (1990) The synapsins. Annu Rev Cell Biol 6:433-460.

De Camilli P, Emr SD, McPherson PS, Novick P (1996) Phosphoinositides as regulators in membrane traffic. Science 271:1533-1539.

Fried RC, Blaustein MP (1978) Retrieval and recycling of synaptic vesicle membrane in pinched-off nerve terminals (synaptosomes). J Cell Biol 78:685-700.

Harris KM, Sultan P (1995) Variation in the number, location and size of synaptic vesicles provides an anatomical basis for the non-uniform probability of release at hippocampal CA1 synapses. Neuropharmacology 34:1387-1397.

Henkel AW, Betz WJ (1995) Staurosporine blocks evoked release of FM1-43 but not acetylcholine from frog motor nerve terminals. J Neurosci 15:8246-8258.

Henkel AW, Lübke J, Betz WJ (1996a) FM1-43 dye ultrastructural localization in and release from frog motor nerve terminals. Proc Natl Acad Sci USA 93:1918-1923.

Henkel AW, Simpson LL, Ridge AP, Betz WJ (1996b) Synaptic vesicle movements monitored by fluorescence recovery after photobleaching in nerve terminals stained with FM1-43. J Neurosci 16:3960-3967.

Hirokawa N, Sobue K, Kanda K, Harada A, Yorifuji H (1989) The cytoskeletal architecture of the presynaptic terminal and molecular structure of synapsin I. J Cell Biol 108:111-126.

Heuser JE, Reese TS (1973) Evidence for recycling of synaptic vesicle membrane during transmitter release at the frog neuromuscular junction. J Cell Biol 57:315-344.
Kraszewski K, Mundigl O, Daniell L, Verderio C, Matteoli M, De Camilli $P$ (1995) Synaptic vesicle dynamics in living cultured neurons visualized with CY3-conjugated antibodies directed against the lumenal domain of synaptotagmin. J Neurosci 15:4328-4342.

Landis DM, Hall AK, Weinstein LA, Reese TS (1988) The organization of cytoplasm at the presynaptic active zone of a central nervous system synapse. Neuron 1:201-209.

Malgaroli A, Ting AE, Wendland B, Bergamaschi A, Villa A, Tsien RW, Scheller RH (1995) Presynaptic component of long-term potentiation visualized at individual hippocampal synapses. Science 268:1624-1628.

Matteoli M, Takei K, Perin MS, Südhof TC, De Camilli P (1992) Exoendocytotic recycling of synaptic vesicles in developing processes of cultured hippocampal neurons. J Cell Biol 117:849-861.

Maycox PR, Link E, Reetz A, Morris SA, Jahn R (1992) Clathrin-coated vesicles in nervous tissue are involved primarily in synaptic vesicle recycling. J Cell Biol 118:1379-1388.

Miller TM, Heuser JE (1984) Endocytosis of synaptic vesicle membrane at the frog neuromuscular junction. J Cell Biol 98:685-698.

Mundigl O, Matteoli M, Daniell L, Thomas-Reetz A, Metcalf A, Jahn R, De Camilli P (1993) Synaptic vesicle proteins in early endosomes in cultured hippocampal neurons: differential effects of Brefeldin A in axon and dendrites. J Cell Biol 122:1207-1221.

Mundigl O, Verderio C, Kraszewski K, De Camilli P, Metteoli M (1995) A radioimmunoassay to monitor synaptic vesicle activity in hippocampal neurons in vitro. Eur J Cell Biol 66:246-256.

Navone F, Greengard P, De Camilli P (1984) Synapsin I in nerve terminals: selective association with small synaptic vesicles. Science 226:1209-1211.

Pfeffer SR, Kelly RB (1985) The subpopulation of brain coated vesicles that carries synaptic vesicle proteins contains two unique polypeptides. Cell 40:949-957.

Pieribone VA, Shupliakov O, Brodin L, Hilfiker-Rothenfluh S, Czernik AJ, Greengard P (1995) Distinct pools of synaptic vesicles in neurotransmitter release. Nature 375:493-497.

Rosahl TW, Spillane D, Missler M, Herz J, Selig DK, Wolff JR, Hammer RE, Malenka RC, Südhof TC (1995) Essential functions of synapsins I and II in synaptic vesicle regulation. Nature 375:488-493.

Ruegg UT, Burgess GM (1989) Staurosporine, K-252 and UCN-01: potent but nonspecific inhibitors of protein kinases. Trends Pharmacol Sci 10:218-220.

Ryan TA, Smith SJ (1995) Vesicle pool mobilization during action potential firing at hippocampal synapses. Neuron 14:983-989.

Ryan TA, Reuter H, Wendland B, Schweizer FE, Tsien RW, Smith SJ (1993) The kinetics of synaptic vesicle recycling measured at a single presynaptic boutons. Neuron 11:713-724.

Ryan TA, Smith SJ, Reuter H (1996) The timing of synaptic vesicle endocytosis. Proc Natl Acad Sci USA 93:5567-5571.

Schiavo G, Benfenati F, Poulain B, Rossetto O, Polverino de Laureto P, DasGupta BR, Montecucco C (1992) Tetanus and botulinum-B neurotoxins block neurotransmitter release by proteolytic cleavage of synaptobrevin. Nature 359:832-835.

Schweizer FE, Betz H, Augustine GJ (1995) From vesicle docking to endocytosis: intermediate reactions of exocytosis. Neuron 14:689-696.

Southwick PL, Ernst LA, Tauriello EW, Parker SR, Mujumdar RB, Mujumdar SR, Clever HA, Waggoner AS (1990) Cyanide dye labeling reagents-carboxymethylindocyanine succinimidyl esters. Cytometry 11:418-430.

Stinchcombe JC, Nomoto H, Cutler DF, Hopkins CR (1995) Anterograde and retrograde traffic between the rough endoplasmic reticulum and the Golgi complex. J Cell Biol 131:1387-1401.

Südhof TC (1995) The synaptic vesicle cycle: a cascade of protein-protein interactions. Nature 375:645-653.

Takei K, Mundigl O, Daniell L, De Camilli P (1996) The synaptic vesicle cycle: a single vesicle budding step involving clathrin and dynamin. J Cell Biol 133:1237-1250. 